DOI: $10.1002 /$ adma.201604424

Article type: Communication

\title{
Exciton Diffusion Length and Charge Extraction Yield in Organic Bilayer Solar Cells
}

Bernhard Siegmund*, Muhammad T. Sajjad, Johannes Widmer, Debdutta Ray, Christian Koerner, Moritz Riede, Karl Leo, Ifor D. W. Samuel*, Koen Vandewal*

B. Siegmund, Dr. J. Widmer, Dr. C. Körner, Prof. K. Leo, Prof. K. Vandewal Dresden Integrated Center for Applied Physics and Photonic Materials (IAPP) and Institute for Applied Physics, Technische Universität Dresden, George-Bähr-Straße 1, 01062 Dresden, Germany

E-mail: bernhard.siegmund@iapp.de, koen.vandewal@iapp.de

Dr. M. T. Sajjad, Prof. I. D. W. Samuel

Organic Semiconductor Centre, SUPA, School of Physics \& Astronomy, North Haugh, St Andrews, KY16 9SS, United Kingdom

E-mail:idws@st-andrews.ac.uk

Prof. D. Ray

Currently: Department of Electrical Engineering, I.I.T. Madras, Chennai 600036, India

Prof. M. Riede

Currently: Clarendon Laboratory, Parks Road, Oxford OX1 3PU, United Kingdom

Keywords: exciton diffusion length, charge carrier extraction, organic photovoltaics, bilayer, photocurrent modelling 


\section{Submitted to

Organic light emitting diodes (OLED), solar cells, and photo-detectors have proven their potential to outperform their inorganic counterparts regarding flexibility, light-weight, transparency, scalability, and fabrication costs as demonstrated by the successful comercialisation of OLEDs. Hereby, all organic opto-electronic devices share a series of electrical and optical processes mediated by a Coulombically bound electron-hole pair called an exciton. Driven by concentration gradients, these electrically neutral quasi-particles cover an average distance $\ell_{\mathrm{d}}$ within their lifetime of typically a few nanoseconds by means of Förster or Dexter energy transfer. The so defined exciton diffusion length is one of the critical material parameters determining the performance of organic opto-electronics. Organic photovoltaics and photodetectors with a blended photoactive layer ${ }^{[1]}$ benefit from a long $\ell_{\mathrm{d}}$ : As the absorber phase separation can be increased, charge recombination is reduced, resulting in an increased operating voltage. ${ }^{[2]}$ Meanwhile, photo-sensitive devices with a planar photoactive structure such as multilayer cascades ${ }^{[3]}$ benefit from a long $\ell_{\mathrm{d}}$ resulting in enhanced short-circuit currents as excitons generated further away from the charge dissociating heterojunction are harvested. For OLEDs on the other hand, a short $\ell_{\mathrm{d}}$ for singlet excitons is required when containing both (efficient) phosphorescent and (stable) fluorescent emitter layers to confine singlet excitons to the latter. ${ }^{[4]}$

In amorphous or polycrystalline organic semiconductors, the diffusion length of singlet excitons is often $10 \mathrm{~nm}$ or less as a result of exciton trapping. ${ }^{[5,6]}$ However, reliability and comparability of $\ell_{\mathrm{d}}$ measurements still remain an issue, even within one technique. ${ }^{[7-9]}$ For example, for the thermally stable and chemically inert small molecule zinc phthalocyanine (ZnPc), reported $\ell_{\mathrm{d}}$-values range over one order of magnitude (see Table $\mathrm{S} 1$ in the Supplementary Information (SI)). Many of these differences can be attributed to a discrepancy upon modelling of the photo-current, as discussed further in Section SI.1. Therefore, this article provides a refined technique based on modelling photocurrent spectra of solar cells 

comprising the material of interest. Our method gives consistent results even for rigorously differing samples architectures and the obtained diffusion length agrees well with complementary measurements based on photoluminescence (PL) quenching.

In contrast to PL based techniques, electrical measurements also allow to study $\ell_{\mathrm{d}}$ of nonfluorescent materials. A key advantage of our photovoltaic approach is the simultaneous quantitative access to both the exciton diffusion length and the yield of charge extraction. To account for potential losses during the splitting of excitons at the donor-acceptor (DA) interface or the subsequent charge transit to the desired electrode, via recombination processes, we introduce a charge collection yield $\eta_{\mathrm{c}}$. It mediates between exciton diffusion towards the DA-interface, leading to a generation flux of CT states $\varphi_{\mathrm{CT}}\left(\ell_{\mathrm{d}}\right)$, and the extracted photocurrent density $j_{\text {photo }}$ via

$$
\mathrm{j}_{\text {photo }}=\mathrm{e} \eta_{\mathrm{c}} \varphi_{\mathrm{CT}}\left(\ell_{\mathrm{d}}\right)
$$

with $e$ as elementary charge. ${ }^{[10]}$ The value of $\eta_{\mathrm{c}}$ is often hard to determine, both in experiment and simulation (details in SI.2). This uncertainty affects the accuracy of $\ell_{\mathrm{d}}$ when determining it using Equation 1. On the other hand, for known exciton dynamics, steady-state or transient ${ }^{[11]}$ measurements of $j_{\text {photo }}$ seem a suitable choice to access $\eta_{\mathrm{c}}$. In this study, however, we demonstrate access to $\ell_{\mathrm{d}}$ and $\eta_{\mathrm{c}}$ at the same time, based on easy and standardised methods, namely measurements of photovoltaic external quantum efficiencies (EQE) and thin-film optics. For this purpose, we utilise a simultaneous variation of the layer thickness $x_{\mathrm{o}}$ and the excitation wavelength $\lambda$ in conjunction with optical simulations.

In our exemplary study of $\mathrm{ZnPc}$, we build pin-type bilayer solar cells with $\mathrm{C}_{60}$ as efficient quencher for $\mathrm{ZnPc}$ excitons. ${ }^{[12]}$ This allows characterising the material in an application oriented layer sequence, as often used in organic solar cells (alternative exciton quenchers for 
high band-gap absorbers are discussed in SI.3a). A first set of solar cells comprises as electron transport layer (ETL) $\mathrm{C}_{60}$ n-doped with $\mathrm{W}_{2}(\mathrm{hpp})_{4}$ (chemical nomenclatures in Experimental Section) and as hole transport layer (HTL) BF-DPB p-doped with $\mathrm{C}_{60} \mathrm{~F}_{36}$. As shown in Figure 1 (top), we center the maximum of the optical field in the ZnPc layer for $680 \mathrm{~nm}$, a wavelength close to which $\mathrm{ZnPc}$ has its peak absorption (details in SI.3b). For centering, we adjust the thickness of HTL and ETL for all ZnPc thicknesses $x_{\mathrm{o}}$ by means of numerical transfer-matrixsimulations $^{[13]}$.

As shown in Figure 2, the measured EQE maximises for a $\mathrm{ZnPc}$ thickness $x_{\mathrm{p}}$ between $15 \mathrm{~nm}$ and $18 \mathrm{~nm}$, increasing with $\lambda$. This already indicates $\ell_{\mathrm{d}}$ to be much smaller than the absorption length which for $\mathrm{ZnPc}$ is above $100 \mathrm{~nm}$. For modelling the measured photocurrent data as function of $x_{\mathrm{o}}$ and $\lambda$, we consider the general exciton diffusion equation

$$
\frac{\partial n}{\partial t}=D \frac{\partial^{2} n}{\partial x^{2}}+G(x, t)-k_{P L}(t) \cdot n-k_{F R E T}(x) \cdot n-\alpha \cdot n^{2}
$$

where $n$ denotes the density distribution of singlet excitons at position $x$ in the absorber film (here $\mathrm{ZnPc}$ ), $D$ the diffusion coefficient and $G(x, t)$ the time dependent exciton generation profile. The radiative decay rate in absence of quencher sites is $k_{\mathrm{PL}}$, whereas $k_{\mathrm{FRET}}$ denotes the rate of Förster resonance energy transfer (FRET) in presence of a neighbouring material with equal or smaller optical gap. $\alpha$ quantifies the rate for exciton-exciton-annihilation.

Exciting the samples under steady-state conditions and low intensities (translating to exciton densities far below the annihilation threshold ${ }^{[14]}$ of $10^{18} \mathrm{~cm}^{-3}$ ) permits to apply Equation 2 with the following simplifications: $\partial n / \partial t=0, k_{\mathrm{PL}}=\tau_{\mathrm{o}}{ }^{-1}=\mathrm{D} / \ell_{\mathrm{d}}{ }^{2}, \alpha=0$ with $\tau_{\mathrm{o}}$ as intrinsic exciton lifetime. Considering only low energy photons $(\lambda>635 \mathrm{~nm}$, see SI.3b) allows to draw exclusive conclusions about the exciton dynamics in $\mathrm{ZnPc}$, due to its smaller optical bandgap $(1.5 \mathrm{eV})$ in 
Submitted to

\section{ADVANCED}

comparison to $\mathrm{C}_{60}(1.8 \mathrm{eV})$. Furthermore, embedding $\mathrm{ZnPc}$ into media $m$ of a greater optical gap, $p$-BF-DPB with $3.0 \mathrm{eV}$ and $\mathrm{C}_{60}$, allows neglecting FRET dynamics and thus circumvents additional PL-studies to determine the Förster radius ${ }^{[15]}: k_{\mathrm{FRET}}^{\mathrm{ZnPc}, \mathrm{m}} \approx 0$. Moreover, within the framework of interference based thin-film-optics ${ }^{[13]}$, we find the spatial distribution of the optical field, and thus the exciton generation, to be approximately parabolic $G_{\lambda}(x) \approx a_{\lambda} x^{2}+b_{\lambda} x+c_{\lambda}$ within a single layer (details in SI.4). As boundary conditions for our simplified diffusion equation

$$
a_{\lambda} x^{2}+b_{\lambda} x+c_{\lambda}=D\left(\frac{1}{\ell_{d}^{2}}-\frac{\partial^{2}}{\partial x^{2}}\right) n
$$

we choose $\mathrm{ZnPc}$ excitons reaching the HTL to be reflected $(\partial n / \partial x(\mathrm{x}=\mathrm{ZnPc} \mid \mathrm{HTL})=0$, details in SI.5) and those reaching $\mathrm{C}_{60}$ to decay immediately into charge transfer $(\mathrm{CT})$ states ${ }^{[16]}$ $\left(n\left(x=\mathrm{ZnPc} \mid \mathrm{C}_{60}\right)=0\right)^{[12]}$.

We obtain an analytical expression for the photo-current as a function of $\ell_{\mathrm{d}}$ and $\eta_{\mathrm{c}}$, derived as Equation S11 in SI.6. We apply it to simultaneously fit all EQE spectra (solid lines) under variation of $\lambda$ and $x_{0}$, as representatively depicted in Figure 2 for four wavelengths of the analysed interval between $635 \mathrm{~nm}$ and $800 \mathrm{~nm}$ (exclusive $\mathrm{ZnPc}$ absorption). For this purpose, $\eta_{c}$ is assumed to be independent of $\lambda$, as wavelength independent internal quantum efficiencies have been experimentally demonstrated for both polymer and small molecule systems. ${ }^{[2,17]}$ We exclude thin ZnPc layers up to a threshold thickness of $x_{\mathrm{t}}=11 \mathrm{~nm}$ from the fitting routine due to layer roughness ${ }^{[18]}$ and DA inter-diffusion ${ }^{[8,19]}$ (details in SI.6b). For thicker absorber layers, we model the charge extraction to be spatially uniform, as no significant concentration of recombination centers is present in the bulk of the neat absorber. $^{[20-22]}$ 
Following this procedure unfolds an exciton diffusion length in $\mathrm{ZnPc}$ of $\ell_{\mathrm{d}}=(10.0 \pm 0.8) \mathrm{nm}$ and a collection yield of $\eta_{\mathrm{c}}=(58 \pm 6) \%$. Note that a successful EQE fit features a peak position $x_{\mathrm{p}}$ coinciding with the experimental data (Figure 2). As the optical field is constructed to be approximately constant in $\mathrm{ZnPc}, x_{\mathrm{p}}$ is found in proximity to the diffusion length. However, for smaller $\ell_{\mathrm{d}}$ it would be desirable to achieve a peak position $x_{\mathrm{p}}$ much greater than the diffusion length to still overcome the thickness threshold for uniform charge extraction $\left(\mathrm{x}_{\mathrm{p}}>\mathrm{x}_{\mathrm{t}}\right)$.

For this purpose, we introduce a pronounced slope in the optical field in the absorber rising with distance to the quenching interface. It aims to extend the exciton diffusion towards this interface, at the cost of overall exciton density. A second set of solar cells (Figure 1 bottom) is prepared showing such a field gradient in absence of thicknesses variations of both transport layers. It also differs with respect to its electronic polarity through inverse layer stacking and its transport layers. Here, BPhen is used as ETL and as HTL MeO-TPD (optical gap of 2.9eV) p-doped with $\mathrm{F}_{6}$-TCNNQ. Comparing to set 1 , we observe a pronounced shift of the EQE peak position to $x_{\mathrm{p}} \gtrsim 25 \mathrm{~nm}$ (see Figure S3 in SI.7). Taking the respective field distribution into account and assuming complete exciton reflection at the interface between $\mathrm{ZnPc}$ and intrinsic MeO-TPD, we obtain $\ell_{d}=(10.2 \pm 0.8) \mathrm{nm}$ in $\mathrm{ZnPc}$ and a collection yield of $\eta_{\mathrm{c}}=(58 \pm 6) \%$ which confirms the previous results within the measurement uncertainty.

The agreement of the fitted values proves the modelling approach to be independent of the considered device geometry and underlines the thickness of maximum photo-current $x_{\mathrm{p}}$ typically not be identical with $\ell_{\mathrm{d}}$. Furthermore, the result implies equally efficient charge extraction for the transport layers of set 1 and 2, as expected. For both device architectures, CT-states at the DA-interface formed upon successful exciton diffusion are inefficiently harvested as extracted photo-current. This can be attributed to incomplete CT dissociation and has been observed in $\mathrm{ZnPc}-\mathrm{C}_{60}$-cells before by Rand et al. ${ }^{[23]}$ The authors attributed this 
lecules with regard to the interface with finding to an unfavourable orientation of the $\mathrm{ZnPc}$ molecules with regard to the interface with $\mathrm{C}_{60}$. Beyond, the measurements recorded in such an exciton diffusion study can be used to evaluate the potential of a corresponding blended absorber system as discussed in SI.8.

To obtain an independent confirmation of the outlined method, an alternative method to determine $\ell_{\mathrm{d}}$ based on the transient decay of PL is performed. ${ }^{[8,24]}$ A sequence of ZnPc thinfilms with varying layer thickness is prepared on quartz glass substrates in the absence and presence of $50 \mathrm{~nm} \mathrm{C}_{60}$, as shown in Figure 3 (top). The $\mathrm{ZnPc} \mathrm{C}_{60}$-interface acts as a complete quencher ${ }^{[12]}$ and reduces the effective exciton lifetime. Meanwhile, ZnPc excitons at the interface with quartz glass or vacuum are expected to reflect, with vacuum being known as an imperfectly reflecting medium. ${ }^{[25]}$ For a conjoint analysis (details in SI.9) of both thin-film sets, a remarkably good agreement is obtained between all measured and modelled PL decays (Figure 3 bottom). The diffusion length $\ell_{\mathrm{d}}=(9.6 \pm 0.8) \mathrm{nm}$ is obtained using a Förster radius of $(1.4 \pm 0.4) \mathrm{nm}$, and agrees well with the value obtained via spectral modelling, presented earlier. As the interface between $\mathrm{ZnPc}$ and vacuum is modelled to completely reflect excitons, it yields the possibility of a slight underestimation of $\ell_{\mathrm{d}}$.

In summary, we have demonstrated an experimentally easily accessible approach to determine the singlet exciton diffusion length $\ell_{\mathrm{d}}$. We demonstrate its validity using $\mathrm{ZnPc}$ as an example but believe it to be applicable to a wider range of organic absorbers. The analysis involves i) preparing a series of bilayer solar cells with varying absorber thickness, ii) measuring their external quantum efficiency spectra, and iii) evaluating the respective device optics. The approach allows the determination of the exciton diffusion length and also the product of the combined yield of charge-transfer state dissociation with subsequent charge extraction $\eta_{\mathrm{c}}$. For $\mathrm{ZnPc}-\mathrm{C}_{60}$ as photoactive system, this procedure yields an exciton diffusion length in $\mathrm{ZnPc} \ell_{\mathrm{d}}$ of $(10.1 \pm 0.9) \mathrm{nm}$ and $\eta_{\mathrm{c}}=(58 \pm 6) \%$. Two rigorously differing solar cell architectures were 
furthermore in excellent agreement with

studied and give consistent results. The value of $\ell_{\mathrm{d}}$ is furthermore in excellent agreement with time resolved measurements of photoluminescence quenching resulting in a diffusion length $\ell_{\mathrm{d}}$ of $(9.6 \pm 0.8) \mathrm{nm}$, which underlines the reliability of the newly proposed method.

\section{Experimental Section}

Preparation of solar cells: Substrates with pre-structured indium tin oxide (ITO, 90nm, $32 \Omega / \square$, Thin Films Devices, USA) are purchased as bottom electrode. All organic and metal layers are deposited via thermal evaporation in ultra high vacuum (K. J. Lesker, UK) with a base pressure of around $10^{-8}$ mbar. Quartz crystal micro-balances are used to monitor the deposition rates (for organics $0.3-0.4 \AA$ /s) and thus to control layer thicknesses and doping ratios. Set 1 follows nip-type layer sequence (40nm- $\left.\mathrm{x}_{0} / 2\right)$ of $\mathrm{C}_{60} \mathrm{n}$-doped with 3 weight(wt)\% Tetrakis(1,3,4,6,7,8-hexahydro-2H-pyrimido[1,2-a]pyrimidinato)ditungsten(II) $\quad\left(\mathrm{W}_{2}(\mathrm{hpp})_{4}\right)$, $20 \mathrm{~nm}$ of intrinsic $\mathrm{C}_{60}, x_{\mathrm{o}}$ of $\mathrm{ZnPc}$ (Supplier: TCI Europe N.V., Belgium), $\left(82 \mathrm{~nm}-x_{\mathrm{o}} / 2\right)$ of N4,N4'-bis(9,9-dimethyl-9H-fluoren-2-yl)-N4,N4'-diphenylbiphenyl-4,4'-diamine (BF-DPB) p-doped with $10 \mathrm{wt} \% \mathrm{C}_{60} \mathrm{~F}_{36}$, and $100 \mathrm{~nm}$ of aluminium (Al) as reflecting top electrode (photoactive area of $6.4 \mathrm{~mm}^{2}$ ). Set 2 follows the pin-type sequence $1 \mathrm{~nm}$ of dopant 2,2'(perfluoronaphthalene-2,6-diylidene)dimalononitrile $\quad\left(\mathrm{F}_{6}\right.$-TCNNQ), 20nm of $\mathrm{N}, \mathrm{N}, \mathrm{N}^{\prime}, \mathrm{N}^{\prime}-$ tetrakis(4-methoxyphenyl)-benzidine (MeO-TPD) p-doped with 2wt\% $\mathrm{F}_{6}$-TCNNQ, 5nm of intrinsic MeO-TPD, $x_{\mathrm{o}}$ of $\mathrm{ZnPc}, 20 \mathrm{~nm}$ of $\mathrm{C}_{60}$, 6nm of 4,7-diphenyl-1,10-phenanthroline (BPhen) and 100nm of Al. Besides the $\mathrm{n}$-dopant $\mathrm{W}_{2}(\mathrm{hpp})_{4}$, all organic materials are purified at least once by vacuum gradient sublimation. After deposition, both sets are sealed under nitrogen with a cover glass and a humidity getter via a UV-hardened epoxy glue.

Analysis of solar cells: For measuring the EQE, xenon light (Oriel Xe Arc-Lamp Apex Illuminator, Newport, US) is filtered to $5 \mathrm{~nm}$ spectral width with a monochromator (Cornerstone 260 1/4 m Monochromator, Newport, US). Excitation light chopped at 215Hz excites the sample on a mask area of $2.8 \mathrm{~mm}^{2} . j_{\text {photo }}$ is detected via a lock-in amplifier (Signal 


\section{Submitted to

Recovery SR 7265, National Instruments, US). A calibrated silicon diode (S1337, Hamamatsu,

Japan) monitors the excitation intensity. A compact CCD spectrometer (USB4000, Ocean Optics, US) reads out the relative excitation spectrum. The optical constants are determined via transmission and reflection (UV-3100 Spectrometer, Shimadzu, Japan) of thin films with varying layer thickness and subsequent optical modelling.

Preparation of PL quenching samples: The same evaporation system is used to deposit thin films of $\mathrm{ZnPc}$ and optionally $\mathrm{C}_{60}$ onto quartz substrates. Storing and analysis of the sample are performed under vacuum.

Analysis of PL quenching samples: Samples are excited with $100 \mathrm{fs}$ pulses at 400nm from a frequency doubled Ti:Sapphire laser. The subsequent decay of the PL is detected between $810 \mathrm{~nm}$ and $830 \mathrm{~nm}$ with a time resolution of $\sim 2 \mathrm{ps}$ via a streak camera (C6860, Hamamatsu, Japan) in synchroscan mode with the laser at $80 \mathrm{MHz}$. 


\section{Supporting Information}

\section{Submitted to

Supporting Information is available from the Wiley Online Library or from the author.

\section{Acknowledgements}

We thank the German BMBF for funding within the scope of the projects InnoProfile 2.2 (03IPT602X) and MEDOS (03EK3503A) as well as the European Commission within the scope of Career Integration Grant (FP7, MSCA, 630864). We also thank Caroline Walde and Andreas Wendel from IAPP for sample preparation as well as Dr. Mauro Furno and Sim4tec for providing the software for optical transfer matrix calculations. I.D.W.S. and M.T.S. acknowledge support from the European Research Council (grant number 321305). I.D.W.S. also acknowledges a Royal Society Wolfson Research Merit Award. K.L. is a fellow of the Canadian Institute for Advanced Research (CIFAR).

Received: ..

Revised:

Published online: 
Submitted to

[1] R. Meerheim, C. Körner, B. Oesen, K. Leo, Appl. Phys. Lett. 2016, 108, 103302.

[2] K. Vandewal, S. Albrecht, E. T. Hoke, K. R. Graham, J. Widmer, J. D. Douglas, M. Schubert, W. R. Mateker, J. T. Bloking, G. F. Burkhard, A. Sellinger, J. M. J. Fréchet, A. Amassian, M. K. Riede, M. D. McGehee, D. Neher, A. Salleo, Nat. Mater. 2014, 13, 63.

[3] K. Cnops, B. P. Rand, D. Cheyns, B. Verreet, M. A. Empl, P. Heremans, Nat. Commun. 2014, 5, 3406.

[4] Y. Sun, N. C. Giebink, H. Kanno, B. Ma, M. E. Thompson, S. R. Forrest, Nature 2006, $440,908$.

[5] S. Athanasopoulos, E. Hennebicq, D. Beljonne, A. B. Walker, J. Phys. Chem. C 2008, 112,11532 .

[6] O. V. Mikhnenko, M. Kuik, J. Lin, N. Van Der Kaap, T.-Q. Q. Nguyen, P. W. M. Blom, Adv. Mater. 2014, 26, 1912.

[7] J. D. A. Lin, O. V. Mikhnenko, J. Chen, Z. Masri, A. Ruseckas, A. Mikhailovsky, R. P. Raab, J. Liu, P. W. M. Blom, M. A. Loi, C. J. García-Cervera, I. D. W. Samuel, T.-Q. Nguyen, Mater. Horizons 2014, 1, 280.

[8] P. E. Shaw, A. Ruseckas, I. D. W. Samuel, Adv. Mater. 2008, 20, 3516.

[9] M. T. Sajjad, A. J. Ward, C. Kästner, A. Ruseckas, H. Hoppe, I. D. W. Samuel, J. Phys. Chem. Lett. 2015, 6, 3054.

[10] A. K. Ghosh, T. Feng, J. Appl. Phys. 1978, 49, 5982.

[11] T. K. Mullenbach, R. J. Holmes, Appl. Phys. Lett. 2015, 107, 123303.

[12] Y. Terao, H. Sasabe, C. Adachi, Appl. Phys. Lett. 2007, 90, 103515.

[13] L. A. A. Pettersson, L. S. Roman, O. Inganäs, J. Appl. Phys. 1999, 86, 487.

[14] A. J. Lewis, A. Ruseckas, O. P. M. Gaudin, G. R. Webster, P. L. Burn, I. D. W. Samuel, Org. Electron. 2006, 7, 452.

[15] W. A. Luhman, R. J. Holmes, Adv. Funct. Mater. 2011, 21, 764.

[16] K. Vandewal, K. Tvingstedt, A. Gadisa, O. Inganäs, J. V. Manca, Phys. Rev. B 2010, $81,125204$.

[17] K. Vandewal, Z. F. Ma, J. Bergqvist, Z. Tang, E. G. Wang, P. Henriksson, K. Tvingstedt, M. R. Andersson, F. L. Zhang, O. Inganas, Adv. Funct. Mater. 2012, 22, 3480 .

[18] C. Schünemann, C. Elschner, A. A. Levin, M. Levichkova, K. Leo, M. Riede, Thin Solid Films 2011, 519, 3939.

[19] M. Drees, R. M. Davis, J. R. Heflin, Phys. Rev. B 2004, 69, 165320. 


\section{Submitted to \\ ADVANECD
MAATRRALS}

[20] F. Yang, S. R. Forrest, ACS Nano 2008, 2, 1022.

[21] A. Foertig, A. Wagenpfahl, T. Gerbich, D. Cheyns, V. Dyakonov, C. Deibel, Adv. Energy Mater. 2012, 1483.

[22] W. Tress, A. Petrich, M. Hummert, M. Hein, K. Leo, M. Riede, Appl. Phys. Lett. 2011, 98, 063301.

[23] B. P. Rand, D. Cheyns, K. Vasseur, N. C. Giebink, S. Mothy, Y. Yi, V. Coropceanu, D. Beljonne, J. Cornil, J. L. Brédas, J. Genoe, Adv. Funct. Mater. 2012, 22, 2987.

[24] A. J. Ward, A. Ruseckas, I. D. W. Samuel, J. Phys. Chem. C 2012, 116, 23931.

[25] O. V Mikhnenko, F. Cordella, A. B. Sieval, J. C. Hummelen, P. W. M. Blom, M. A. Loi, J. Phys. Chem. B 2009, 9104. 


\section{Submitted to \\ ADVANEDS
MATERRALIS}

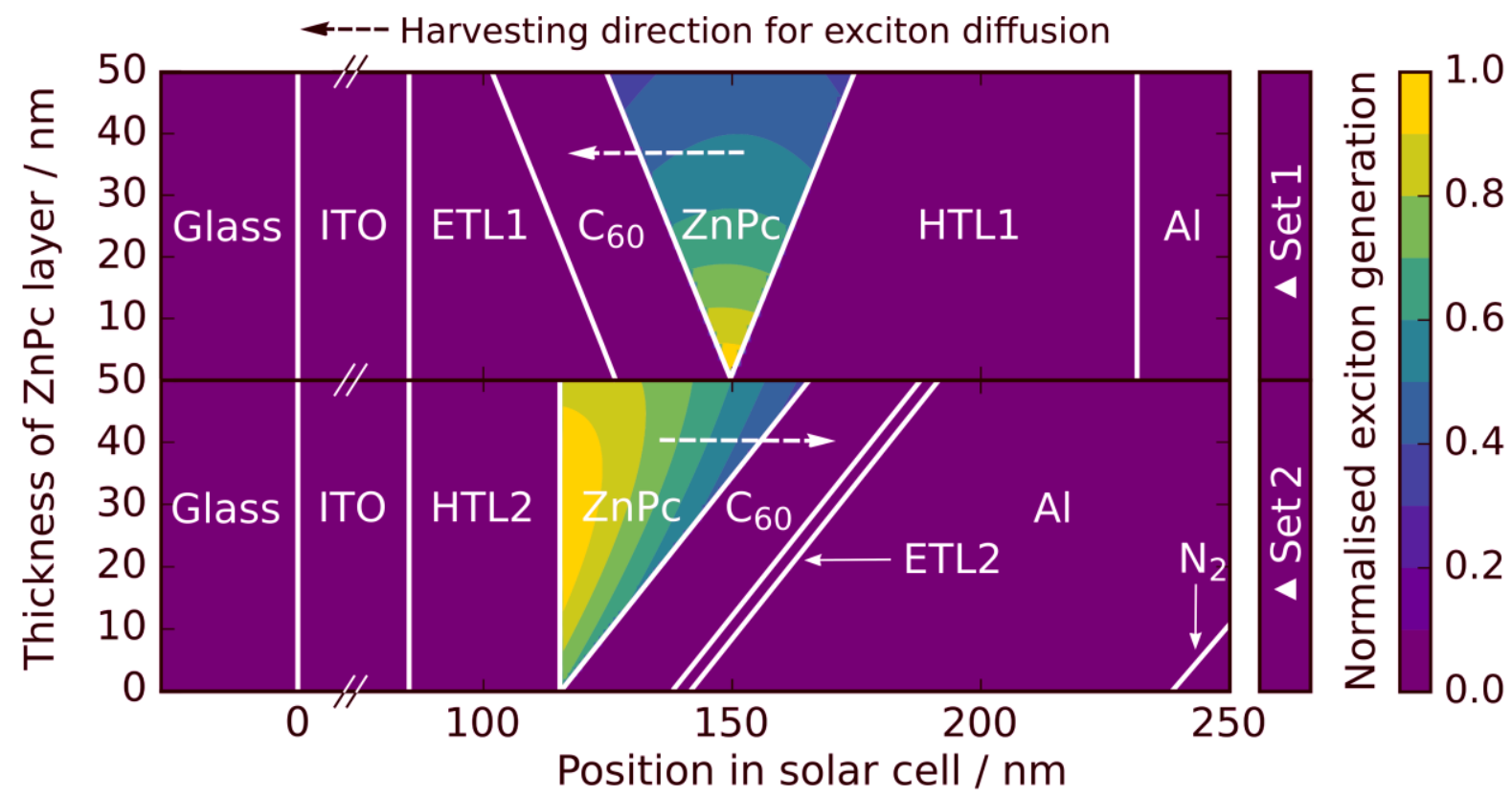

Figure 1. Composition and simulation of the exciton generation profile of the solar cell sets 1 and 2 under variation of the $\mathrm{ZnPc}$ layer thickness $x_{\mathrm{o}}$ when excited with a wavelength of $680 \mathrm{~nm}$. The device sets differ in their i) electronic polarity, ii) choice of transport layers (ETL1=n-doped $\mathrm{C}_{60}$, ETL2=intrinsic BPhen, HTL1=p-doped BF-DPB, HTL2=intrinsic MeOTPD (5nm, touching ZnPc) followed by p-doped MeO-TPD) and iii) optical field distribution in $\mathrm{ZnPc}$ : Each device of set 1 exhibits an approximately homogeneous field, as illustrated for example for the device $x_{0}=24 \mathrm{~nm}$ whose field fluctuations are restricted to only $5 \%$. The optical profiles for solar cells of set 2, in contrast, describe a distinct slope in the ZnPc layer whose amplitude decreases towards the quenching $\mathrm{C}_{60}$ interface, for instance for $x_{0}=24 \mathrm{~nm}$ by about $50 \%$. 


\section{Submitted to

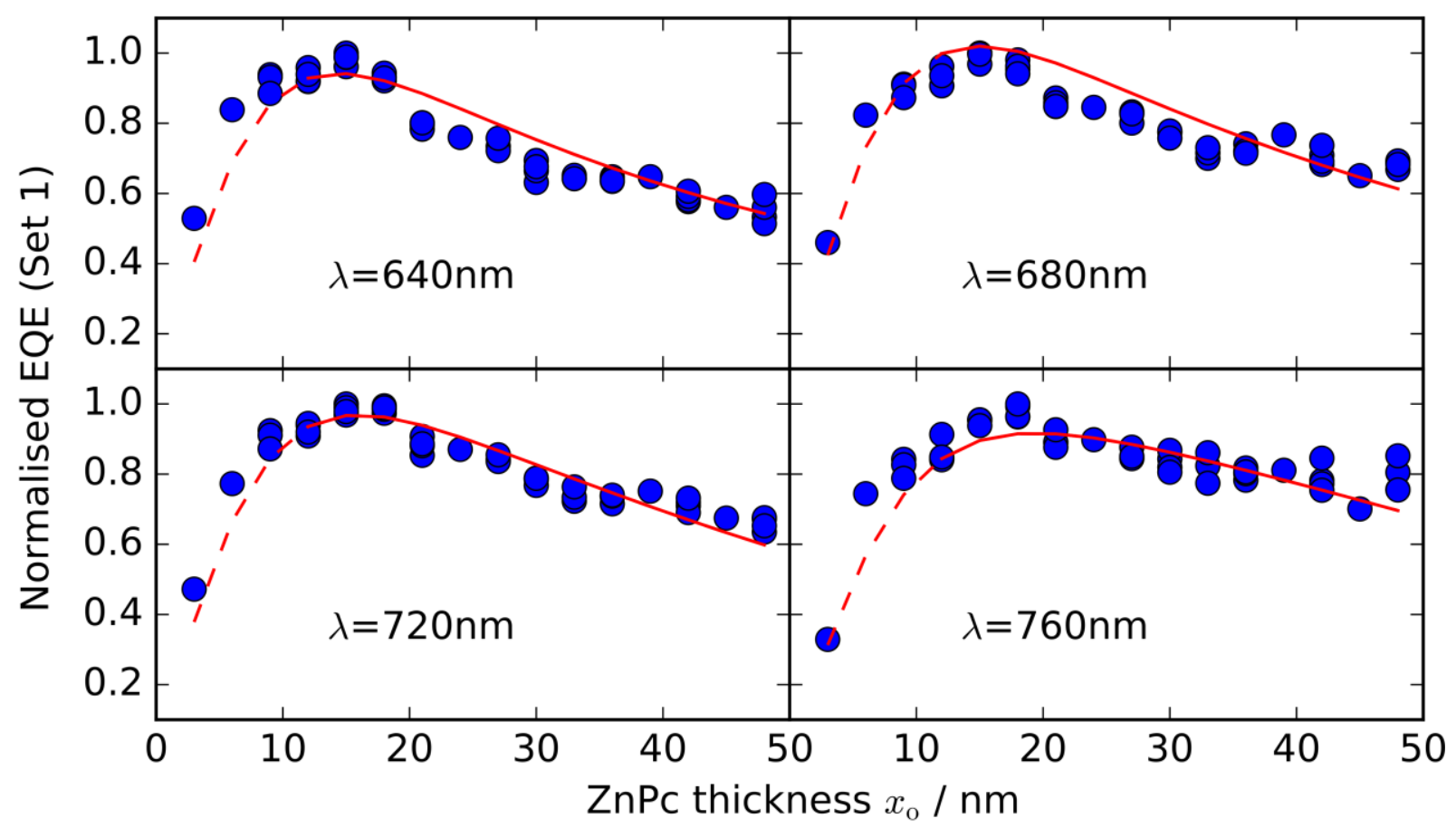

Figure 2. External quantum efficiency (EQE) of set 1 from Figure 1 under variation of the layer thickness of $\mathrm{ZnPc} x_{\mathrm{o}}$ and the excitation wavelength $\lambda$. The experimental data (circles) is jointly fitted (solid lines) for all $x_{\mathrm{o}}$ and $\lambda$ according to Equation S11 in SI.6. The dashed lines indicate the prolongation of the fit for the range of discarded samples $\left(x_{0} \leq 11 \mathrm{~nm}\right)$. The extracted diffusion length in $\mathrm{ZnPc}$ is $\ell_{\mathrm{d}}=(10.0 \pm 0.8) \mathrm{nm}$ and the charge extraction yield is $\eta_{\mathrm{c}}=$ $(58 \pm 6) \%$. For better readability, the EQE is normalised to its experimental maximum for each sub-graph, namely to $18.1 \%, 15.3 \%, 13.4 \%$ and $7.5 \%$ (read for increasing wavelength). 


\section{Submitted to
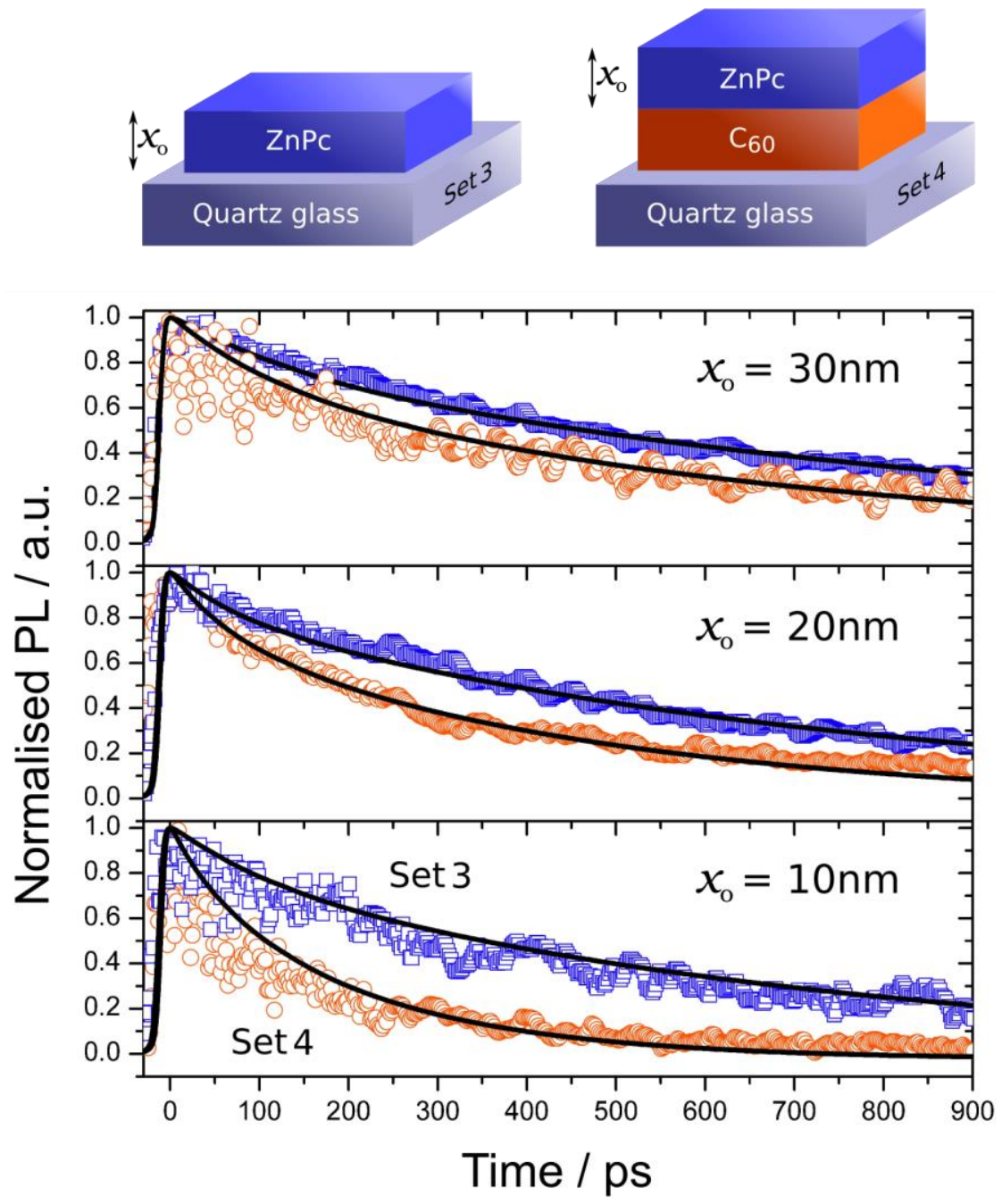

Figure 3. Top: Schematics of the series of investigated thin films with a thickness variation of ZnPc. Bottom: Corresponding experimental time-resolved fluorescence decays measured using a detection wavelength range of $810-830 \mathrm{~nm}$. The wavelength of $400 \mathrm{~nm}$ is used to excite the samples. The bare absorber on quartz glass (set 3, top signal as blue squares) is analysed in conjunction with the corresponding sample with a quenching $\mathrm{C}_{60}$ sublayer (set 4 , bottom signal as orange circles). The best fit (black line) using Equation $2(G=0, \alpha=0)$ is obtained for $\ell_{\mathrm{d}}=(9.6 \pm 0.8) \mathrm{nm}$ and a Förster radius of $(1.4 \pm 0.4) \mathrm{nm}$. 


\section{ToC entry:}

A method for resolving the diffusion length of excitons and the extraction yield of charge carriers is presented based on the performance of organic bilayer solar cells and careful modeling. The technique uses a simultaneous variation of the absorber thickness and the excitation wavelength. Rigorously differing solar cell structures as well as independent photoluminescence quenching measurements give consistent results.

Keyword:

exciton diffusion length, charge carrier extraction, organic photovoltaics, bilayer, photocurrent modelling

B. Siegmund*, M. T. Sajjad, J. Widmer, D. Ray, C. Koerner, M. Riede, K. Leo, I. D. W. Samuel*, K. Vandewal*

Title:

Exciton Diffusion and Charge Carrier Extraction in Organic Bilayer Solar Cells

ToC figure

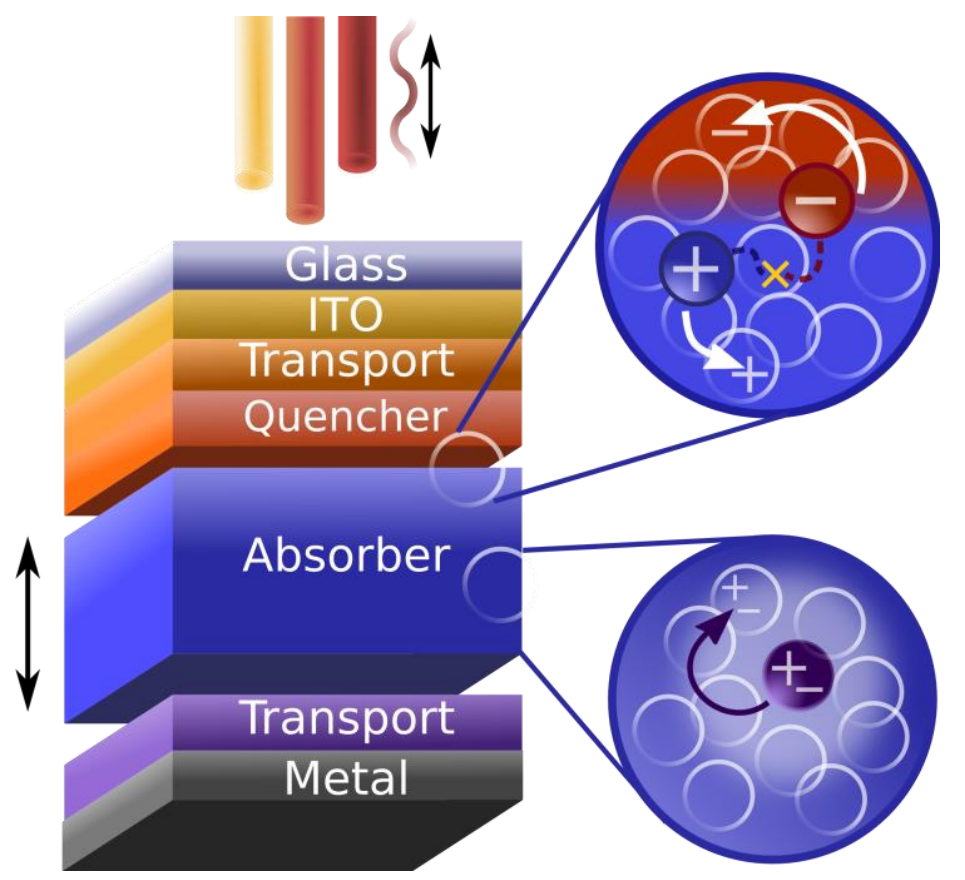

